SPhT T01/25

math-ph/0103010

\title{
Higher-Order Corrections to Instantons
}

\author{
Ulrich D. Jentschura† and Jean Zinn-Justin $\ddagger$ \\ $\dagger$ Laboratoire Kastler-Brossel, Unité Mixte de Recherche du CNRS No. C8552, \\ Université Pierre et Marie Curie, Case 74, 4 pl. Jussieu, F-75252 Paris Cedex 05, France \\ $\ddagger$ Commissariat à l'Énergie Atomique, Service de Physique Théorique, \\ F-91191 Gif-Sur-Yvette Cedex, France \\ Email: ulj@spectro.jussieu.fr, zinn@spht.saclay.cea.fr
}

\begin{abstract}
The energy levels of the double-well potential receive, beyond perturbation theory, contributions which are non-analytic in the coupling strength; these are related to instanton effects. For example, the separation between the energies of odd- and even-parity states is given at leading order by the one-instanton contribution. However to determine the energies more accurately multi-instanton configurations have also to be taken into account. We investigate here the two-instanton contributions. First we calculate analytically higherorder corrections to multi-instanton effects. We then verify that the difference betweeen numerically determined energy eigenvalues, and the generalized Borel sum of the perturbation series can be described to very high accuracy by two-instanton contributions. We also calculate higher-order corrections to the leading factorial growth of the perturbative coefficients and show that these are consistent with analytic results for the two-instanton effect and with exact data for the first 200 perturbative coefficients.
\end{abstract}

PACS numbers 11.15.Bt, 11.10.Jj

Keywords General properties of perturbation theory;

$\overline{\text { Asymptotic problems and properties }}$ 
In theories with degenerate minima, the energy eigenvalues of the states at nonvanishing coupling $g \neq 0$ cannot in principle be obtained by analytic continuation from the unperturbed situation at vanishing coupling $g=0$ because a potential with degenerate minima introduces a degeneracy in the spectrum: for any one unperturbed state, two states emerge when the perturbation is switched on. These two states are seperated by an energy shift which is nonperturbative and nonanalytic in the coupling, i.e. vanishing to any order in perturbation theory. Therefore, the two states are described by the same perturbation series and yet differ in their energy by instanton contributions. Specifically, we consider the case of the double-well potential with the hamiltonian

$$
H=-\frac{g}{2} \frac{\partial^{2}}{\partial q^{2}}+\frac{1}{g} V(q) \quad V(q)=\frac{1}{2} q^{2}(1-q)^{2} .
$$

It has been conjectured [1] that an asymptotic expansion for the energy eigenvalue can be obtained by finding a solution to the equation

$$
\frac{1}{\sqrt{2 \pi}} \Gamma\left(\frac{1}{2}-D(E, g)\right)\left(-\frac{2}{g}\right)^{D(E, g)} \exp [-A(E, g) / 2]= \pm \mathrm{i},
$$

which can be understood as a modified Bohr-Sommerfeld quantization condition. The plus and minus signs apply to even- and odd-parity states, respectively. The conjecture (2), whose validity has been proven in [5], has found a natural explanation in the framework of Ecalle's theory of resurgent functions [6 8]. The functions $D(E, g), A(E, g)$ constitute power series in both variables. The function $D(E, g)$ describes the perturbative expansion; its evaluation is discussed in [1, [4]. The first terms read

$$
D(E, g)=E+g\left(3 E^{2}+\frac{1}{4}\right)+g^{2}\left(35 E^{3}+\frac{25}{4} E\right)+\mathcal{O}\left(g^{2}\right) .
$$

The ground and the first excited state are both described by the same perturbation series which can be found by inverting the equation $D(E, g)=1 / 2$. When the energy is expressed in terms of the naive perturbation series in $g$, the function $D(E, g)$ then vanishes in any order of perturbation theory, i.e. in all orders in $g$. For a general state, $D(E, g)=N+1 / 2$ where $N$ is the quantum number of the unperturbed state which is a harmonic oscillator eigenstate. The function $A(E, g)$ essentially describes instanton contributions 44; its first terms read

$$
A(E, g)=\frac{1}{3 g}+g\left(17 E^{2}+\frac{19}{12}\right)+g^{2}\left(227 E^{3}+\frac{187}{4} E\right)+\mathcal{O}\left(g^{2}\right) \text {. }
$$

A solution to the equation (2) can be found by systematically expanding the energy eigenvalue $E(g)$ in powers of $g$ and in the two quantities

$$
\lambda(g)=\ln \left(-\frac{2}{g}\right) \quad \text { and } \quad \xi(g)=\frac{\exp [-1 /(6 g)]}{\sqrt{\pi g}} .
$$

Terms of order $\xi(g)^{n}$ belong to the $n$-instanton contribution. The energy eigenvalue for nonvanishing perturbation $g \neq 0$ can be described by two quantum numbers: the unperturbed quantum number $N$ and the positive or negative parity of the state. We have (the upper index denotes the instanton order)

$$
E_{N, \pm}(g)=\sum_{n=0}^{\infty} E_{N, \pm}^{(n)}(g)
$$

where the perturbation series (zero-instanton contribution) is given as

$$
E_{N, \pm}^{(0)}(g)=\sum_{K=0}^{\infty} E_{N, K}^{(0)} g^{K}
$$


where the right-hand side is parity independent. For $n>0$, the instanton contribution reads

$$
E_{N, \pm}^{(n)}(g)=\left(\frac{2}{g}\right)^{N n} \xi(g)^{n} \sum_{k=0}^{n-1} \lambda(g)^{k} \sum_{l=0} \epsilon_{n k l}^{(N, \pm)} g^{l} .
$$

The lower indices $n, k$ and $l$ of the $\epsilon$ coefficients denote the instanton order, the power of the logarithm and the power of $g$, respectively. The results relevant for the current investigation read,

$$
\begin{aligned}
& \epsilon_{100}^{(0,+)}=-\epsilon_{100}^{(0,-)}=-1, \quad \epsilon_{101}^{(0,+)}=-\epsilon_{101}^{(0,-)}=\frac{71}{12}, \\
& \epsilon_{101}^{(0,+)}=-\epsilon_{101}^{(0,-)}=\frac{6299}{288}, \quad \epsilon_{210}^{(0,+)}=\epsilon_{210}^{(0,-)}=1, \\
& \epsilon_{211}^{(0,+)}=\epsilon_{211}^{(0,-)}=-\frac{53}{6}, \quad \epsilon_{212}^{(0,+)}=\epsilon_{212}^{(0,-)}=-\frac{1277}{72}, \\
& \epsilon_{200}^{(0,+)}=\epsilon_{200}^{(0,-)}=\gamma, \quad \epsilon_{201}^{(0,+)}=\epsilon_{201}^{(0,-)}=-\frac{23}{2}-\frac{53}{6} \gamma, \\
& \epsilon_{202}^{(0,+)}=\epsilon_{202}^{(0,-)}=\frac{13}{12}-\frac{1277}{72} \gamma,
\end{aligned}
$$

where $\gamma=0.57221 \ldots$ is Euler's constant. Odd-instanton contributions have opposite sign for oppositeparity states and are responsible, in particular, for the energy difference of the ground state with quantum numbers $(0,+)$ and the first excited state with quantum numbers $(0,-)$. The dominant contribution to the seperation of the two lowest energy levels is given by the one-instanton contribution:

$$
E_{0,-}(g)-E_{0,+}(g) \sim 2 \xi(g)\left(1-\frac{71}{12} g-\frac{6299}{288} g^{2}+\mathcal{O}\left(g^{3}\right)\right)+\mathcal{O}\left(\xi(g)^{3}\right) .
$$

By contrast, even-instanton contributions have like sign for opposite-parity states and are responsible, in particular, for the displacement of the mean value $(1 / 2)\left[E_{0,-}(g)+E_{0,+}(g)\right]$ from the value of the generalized Borel sum of the perturbation series $\mathcal{B}\left(\sum_{K=0}^{\infty} E_{0, K}^{(0)} g^{K}\right)$ (for the evaluation of the generalized Borel sum of a nonalternating divergent series see for example Sec. VI and Table III of [9]). The dominant contribution to the displacement comes from the two-instanton effect, and we have

$$
\begin{aligned}
& \frac{1}{2}\left[E_{0,-}(g)+E_{0,+}(g)\right]-\operatorname{Re}\left\{\mathcal{B}\left(\sum_{K=0}^{\infty} E_{0, K}^{(0)} g^{K}\right)\right\} \sim \xi(g)^{2}\left\{\ln \left(\frac{2 \mathrm{e}^{\gamma}}{g}\right)\right. \\
& \left.\quad+g\left[-\frac{53}{6} \ln \left(\frac{2 \mathrm{e}^{\gamma}}{g}\right)-\frac{23}{2}\right]+g^{2}\left[-\frac{1277}{72} \ln \left(\frac{2 \mathrm{e}^{\gamma}}{g}\right)+\frac{13}{12}\right]+\mathcal{O}\left(g^{3} \ln (g)\right)\right\}+\mathcal{O}\left(\xi(g)^{4}\right) .
\end{aligned}
$$

The function [2]

$$
\Delta(g)=4 \frac{\frac{1}{2}\left[E_{0,-}(g)+E_{0,+}(g)\right]-\operatorname{Re}\left\{\mathcal{B}\left(\sum_{K=0}^{\infty} E_{0, K}^{(0)} g^{K}\right)\right\}}{\left[E_{0,-}(g)-E_{0,+}(g)\right]^{2} \ln \left(\frac{2 \mathrm{e}^{\gamma}}{g}\right)} .
$$

relates the multi-instanton contributions to the energy eigenvalues, which can be evaluated numerically, and to the (generalized) Borel sum of the perturbation series which is evaluated by analytic continuation of the integration path into the complex plane (see [10]). The calculation of $\Delta(g)$ at small coupling is 
problematic because of severe numerical cancellations. From the equations (9), (10) and (11), we obtain the following asymptotics for $\Delta(g)$,

$$
\begin{array}{r}
\Delta(g) \sim 1+g\left[\frac{71}{6}+\left(-\frac{53}{6} \ln \left(\frac{2 \mathrm{e}^{\gamma}}{g}\right)-\frac{23}{2}\right) / \ln \left(\frac{2 \mathrm{e}^{\gamma}}{g}\right)\right] \\
+g^{2}\left[\frac{10711}{72}+\left(\frac{1277}{72} \ln \left(\frac{2 \mathrm{e}^{\gamma}}{g}\right)-\frac{13}{12}\right) / \ln \left(\frac{2 \mathrm{e}^{\gamma}}{g}\right)\right]+\mathcal{O}\left(g^{3}\right) .
\end{array}
$$

If we additionally perform an expansion in inverse powers of $\ln (2 / g)$ and keep only the first few terms in $\{1 / \ln (2 / g)\}$ in each term in the $g$-expansion, the result reads

$$
\begin{aligned}
\Delta(g) & \sim 1+3 g-\frac{23}{2} \frac{g}{\ln (2 / g)}\left[1-\frac{\gamma}{\ln (2 / g)}+\frac{\gamma^{2}}{\ln ^{2}(2 / g)}+\mathcal{O}\left(\frac{1}{\ln ^{3}(2 / g)}\right)\right] \\
& +\frac{53}{2} g^{2}-135 \frac{g^{2}}{\ln (2 / g)}\left[1-\frac{\gamma}{\ln (2 / g)}+\frac{\gamma^{2}}{\ln ^{2}(2 / g)}+\mathcal{O}\left(\frac{1}{\ln ^{3}(2 / g)}\right)\right]+\mathcal{O}\left(g^{3}\right) .
\end{aligned}
$$

The higher-order corrections, which are only logarithmically suppressed with respect to the leading terms $1+3 g$, change the numerical values quite significantly, even at small coupling. In Table 11 we present numerical results for the function $\Delta(g)$ at small coupling; these are in agreement with the first few asymptotic terms listed in equation (12) up to numerical accuracy. Of course, for strong coupling, significant deviations from the leading asymptotics must be expected due to higher-order effects; these are indeed observed. For example, at $g=0.1$ the numerically determined value reads $\Delta(0.1)=0.87684(1)$ whereas the first asymptotic terms given in equation (12) sum up to a numerical value of 0.86029 .

Table 1: Comparison of numerical values for the function $\Delta(g)$ defined in equation (11) in the region of small coupling to values obtained by calculating the first few terms in its asymptotic expansion given in (12).

\begin{tabular}{cllllll}
\hline \hline coupling $g$ & 0.005 & 0.006 & 0.007 & 0.008 & 0.009 & 0.010 \\
\hline$\Delta(g)$ num. & $1.0063(5)$ & $1.0075(5)$ & $1.00832(5)$ & $1.00919(5)$ & $1.00998(5)$ & $1.01078(5)$ \\
$\Delta(g)$ asymp. & 1.00640 & 1.00739 & 1.00832 & 1.00919 & 1.01001 & 1.01078 \\
\hline \hline
\end{tabular}

The higher-order corrections to the two-instanton effect are related to the corrections to the leading factorial growth of the perturbative coefficients. This can be seen by expressing that the imaginary part of the perturbation series, when continued analytically from negative to positive coupling, has to cancel with the imaginary part of the two-instanton contribution which is generated by the logarithms $\ln (-2 / g)$. The corrections of order $g \ln (-2 / g)$ and $g^{2} \ln (-2 / g)$ yield the $1 / K$ - and $1 / K^{2}$-corrections to the leading factorial growth of the perturbative coefficients. From the results for $\epsilon_{21 j}^{(0, \pm)}(j=0,1,2)$ given in equation (8), we obtain

$$
E_{0, K}^{0} \sim-\frac{3^{K+1} K !}{\pi}\left[1-\frac{53}{18} \frac{1}{K}-\frac{1277}{648} \frac{1}{K^{2}}+\mathcal{O}\left(\frac{1}{K^{3}}\right)\right] .
$$

The analytic results should be checked against explicit values of the perturbative coefficients. We have determined the first 200 perturbative coefficents $E_{0, K}^{(0)}(K=0, \ldots, 200)$ of the perturbation in the form of 
rational numbers, i.e. to formally infinite numerical accuracy. This allows to verify the $1 / K-$ and $1 / K^{2}-$ corrections to the leading factorial growth in equation (14) to high accuracy, for example by employing Richardson extrapolation [11]. Using the 160th through the 200th perturbation coefficient as input data for the Richardson algorithm, the coefficients of the leading, of the $1 / K$-subleading and of the $1 / K^{2}$ suppressed corrections are found to be consistent with the analytic results given in equation (14) up to a relative numerical accuracy of $10^{-26}, 10^{-23}$ and $10^{-20}$, respectively. For completeness, we give here the numerical values of the 198 th through the 200 th perturbative coefficients, to 30 decimals. These read:

$$
\begin{aligned}
& E_{0,198}^{(0)}=5.501177696288587935277569438632 \times 10^{464}, \\
& E_{0,199}^{(0)}=3.284453984165780006162191232835 \times 10^{467}, \\
& E_{0,200}^{(0)}=1.970821419309543769795300607410 \times 10^{470} .
\end{aligned}
$$

Values for all 200 coefficients will be presented elsewhere.

It is an interesting consequence of the expansion (5) that the energy difference $\left(E_{0,-}-E_{0,+}\right)$, at small coupling, is described to high accuracy by the one-instanton contribution $(n=1$ in equation (7) ). For $g=0.001$, we obtain to 180 decimals,

$$
\begin{aligned}
E_{0,+}(0.001)= & 0.498995454862109171689130839481921636820947240 \\
& 2080966532932786972201391 \underline{15135285053829445798} \\
& \underline{457599599906739551758472267802813069690601325} \\
& \underline{259437728994365882552444017437127892797899793},
\end{aligned}
$$

whereas

$$
\begin{aligned}
E_{0,-}(0.001)= & 0.498995454862109171689130839481921636820947240 \\
& 2080966532932786972201391 \underline{29839929595580370812} \\
& \underline{277499244848259367436475768328848353551134663} \\
& \underline{063098233151885233080862284780527221010367282} .
\end{aligned}
$$

Decimals which differ in the two energy levels are underlined. The results have been obtained by lattice extrapolation using a modified Richardson algorithm which is constructed according to ideas outlined in [12]. Calculations were performed on IBM RISC/6000 workstations while making extensive use of multiprecision libraries [13 15$]$. We define $\mathcal{P}_{M}(g)$ as the $M$ th partial sum of the one-instanton contribution $E_{0,-}^{(1)}(g)-E_{0,+}^{(1)}(g)$,

$$
\mathcal{P}_{M}(g)=2 \xi(g) \sum_{j=0}^{M} \epsilon_{10 j}^{(0,-)} g^{j} .
$$

Using exact rational expressions for the coefficients $\epsilon_{10 j}^{(0,-)}(j \leq 141)$, we obtain

$$
\begin{aligned}
& \mathcal{P}_{140}(0.001) \times 10^{71}= \\
& 1.4704644541750925013819899644941519815678003500526035283, \\
& 8605333378036605041575193505284182673433993282124674888 \\
& \mathcal{P}_{141}(0.001) \times 10^{71}= \\
& 1.4704644541750925013819899644941519815678003500526035283, \\
& 8605333378036605041575193505284182673433993282124674887 .
\end{aligned}
$$


These values are in excellent agreement with the numerically determined energy difference (see the results presented above in equations (16) and (17))

$$
\begin{aligned}
& {\left[E_{0,-}(0.001)-E_{0,+}(0.001)\right] \times 10^{71}=} \\
& 1.4704644541750925013819899644941519815678003500526035283, \\
& \quad 8605333378036605041575193505284182673433993282124674887 .
\end{aligned}
$$

The first 70 decimals in equations (16) and (17) are the same because the one-instanton contribution is of the order of $1.4 \times 10^{-71}$. The accuracy to which the one-instanton contribution describes the energy difference $E_{0,-}(0.001)-E_{0,+}(0.001)$ is limited by the three-instanton effect which for $g=0.001$ is of the order of $8 \times 10^{-212}$. Note that the two-instanton effect (which for $g=0.001$ is of the order of $4 \times 10^{-142}$ ) does not limit the accuracy to which the one-instanton contribution describes the energy difference because it has the same sign and equal magnitude for opposite-parity states with the same unperturbed quantum number $N$.

We have demonstrated that the behavior of the characteristic function $\Delta(g)$ defined in equation (11) at small coupling is consistent with higher-order corrections to the one- and two-instanton contributions, specifically with the instanton expansion of the energy levels governed by the equations (阿) and (7), with the assumption that the instanton contributions given by equation (7) should be Borel summed, with the explicit results for the higher-order coefficients listed in (8) and the analytically derived asymptotics for the function $\Delta(g)$ given in equation (12). The corrections of relative order $1 / K^{m}$ to the leading factorial growth of the perturbative coefficients - see equation (14) - are consistent with the analytically evaluated $g^{m} \ln (-2 / g)$-corrections to the two-instanton effect and with the explicit values for the first 200 terms in the perturbation series (6). The nonperturbative energy difference $E_{0,-}(g)-E_{0,+}(g)$ at small coupling $g$ is described, to high accuracy, by the one-instanton contribution only.

\section{Acknowledgements}

U.D.J. acknowledges support from the Deutscher Akademischer Austauschdienst (DAAD).

\section{References}

[1] J. Zinn-Justin, J. Math. Phys. 22, 511 (1981).

[2] J. Zinn-Justin, Nucl. Phys. B 192, 125 (1981).

[3] J. Zinn-Justin, Nucl. Phys. B 218, 333 (1983).

[4] J. Zinn-Justin, J. Math. Phys. 25, 549 (1984).

[5] E. Delabaere and H. Dillinger, Ph.D. thesis, University of Nice, Nice, 1991 (unpublished).

[6] F. Pham, C. R. Acad. Sci. Paris 309, 999 (1989).

[7] B. Candelpergher, J. C. Nosmas, and F. Pham, Approche de la Résurgence (Hermann, Paris, 1993).

[8] L. Boutet de Monvel (Ed.), Méthodes Résurgentes (Hermann, Paris, 1994).

[9] U. D. Jentschura, e-print physics/0010038 (Phys. Rev. A, in press).

[10] J. Zinn-Justin, Quantum Field Theory and Critical Phenomena, 3rd ed. (Clarendon Press, Oxford, 1996). 
[11] L. F. Richardson, Phil. Trans. Roy. Soc. London A 226, 229 (1927).

[12] E. J. Weniger, Comput. Phys. Rep. 10, 189 (1989).

[13] D. H. Bailey, A portable high performance multiprecision package, NASA Ames RNR Tech. Rep. RNR-90-022.

[14] D. H. Bailey, ACM Trans. Math. Soft. 19, 288 (1993).

[15] D. H. Bailey, A Fortran-90 based multiprecision system, NASA Ames RNR Tech. Rep. RNR-94-013. 